\title{
Prevalence of comorbidities and secondary health conditions among the Finnish population with spinal cord injury
}

\author{
Susanna Tallqvist $\mathbb{D}^{1 凶}{ }^{凶}$, Anna-Maija Kauppila ${ }^{2}$, Aki Vainionpää $^{3}$, Eerika Koskinen (iD) 4 , Paula Bergman (iD ${ }^{5}$, Heidi Anttila (iD ${ }^{6}$, \\ Harri Hämäläinen ${ }^{7}$, Anni Täckman (iD) ${ }^{8}$, Mauri Kallinen ${ }^{9,10}$, Jari Arokoski ${ }^{7}$ and Sinikka Hiekkala (D) ${ }^{11,12}$ \\ (c) The Author(s) 2021
}

\begin{abstract}
STUDY DESIGN: A cross-sectional study.
OBJECTIVES: To explore the prevalence of comorbidities, secondary health conditions (SHCs), and multimorbidity in the Finnish population with spinal cord injury $(\mathrm{SCl})$.

SETTING: The data were collected from the Finnish Spinal Cord Injury Study (FinSCI). Participants were identified from three SCI outpatient clinics responsible for the lifelong follow-up of persons with $\mathrm{SCl}$ in Finland, $(n=884$ participants, response rate; $50 \%$ ). METHODS: The FinSCl-questionnaire included a question from the National Study of Health, Well-being, and Service (FinSote) for screening 12 comorbidities. The reference data of the general population for that question were received from the Finnish Institute for Health and Welfare. The Spinal Cord Injury Secondary Condition Scale (SCI-SCS) was used to screen 16 SHCs. The data were analysed with univariate testing and multivariable negative binomial regression modelling.

RESULTS: The most common comorbidities were high blood pressure/hypertension (38\%), back problems (28\%), and high cholesterol (22\%). The most common SHCs were joint and muscle pain (81\%), muscle spasms (74\%), chronic pain (71\%), and bowel problems (71\%). The prevalence of comorbidities was highest among persons aged $\geq 76$ years (mean; 2.0; scale range; $0-12$ ). The prevalence of SHCs was highest in the severity of SCl group C1-4 AIS A, B, and C (mean; 8.9; scale range; 0-16).

CONCLUSIONS: Further research on geriatrics in $\mathrm{SCl}$, non-traumatic $\mathrm{SCl}$, and knowledge of the needs of persons with cervical lesion AIS A, B, or C is required, due to the fact that the prevalence of multimorbidity is high in these groups.
\end{abstract}

Spinal Cord (2022) 60:618-627; https://doi.org/10.1038/s41393-021-00704-7

\section{INTRODUCTION}

Persons with spinal cord injury $(\mathrm{SCl})$ can experience several comorbidities and secondary health conditions (SHCs) after $\mathrm{SCl}$. The word "comorbidity" refers to the presence of an additional disease in relation to an index disease in one person [1], whereas a "secondary health condition" is a health problem that is either a direct result of the impairment (like $\mathrm{SCl}$ ) or an indirect factor that is related to the impairment [2]. Arthritis, hypertension, hyperlipidaemia, obesity, depression, diabetes, and alcoholism have been reported as some of the most common comorbidities among persons with $\mathrm{SCl}[3,4]$. Similarly, spasticity, chronic pain, sexual dysfunction, bowel and bladder dysfunction, urinary tract infections, sleep problems, and contractures are often experienced as health problems following $\mathrm{SCl}$ [5-8]. "Comorbidity" and "SHC" can be interpreted as synonyms. Terms are differentiated in order to clarify the interpretation of the results, and with the word "comorbidity" we refer to a disease that is treated or diagnosed by a physician. Persons with $\mathrm{SCl}$ can suffer from $\geq 2$ comorbidities and/or SHCs simultaneously, which is known as a multimorbidity [1]. Based on a recent study, age and lesion characteristics had effects on multimorbidity in the analyses of 15 different SHCs [5].

The presence of comorbidities and/or SHCs is a burden to one's health [1, 2], and places demands on health services [1]. As part of the Finnish Spinal Cord Injury Study (FinSCl) [9], the purpose of this article was to describe the prevalence of comorbidities and SHCs, as well as the prevalence of multimorbidity in the Finnish population with $\mathrm{SCl}$. This article also compared the prevalence of these comorbidities and SHCs in relation to age and gender (later referred to as general characteristics), as well as the severity of $\mathrm{SCl}$, aetiology, and time since injury (later referred to as lesion characteristics). The results of this article are focused on the general characteristics and severity of $\mathrm{SCl}$. The prevalence of comorbidities and SHCs has not yet been studied among the Finnish population with $\mathrm{SCl}$. Furthermore, it is important to understand the status of these health problems because Finland is reforming its health care system and the number of persons with $\mathrm{SCl}$ is increasing $[10,11]$.

\footnotetext{
${ }^{1}$ University of Helsinki, Faculty of Medicine, Helsinki, Finland. ${ }^{2}$ Oulu University Hospital, Department of Medical Rehabilitation/Spinal Cord Injury Outpatient Clinic, Oulu, Finland.

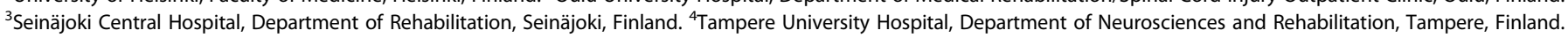

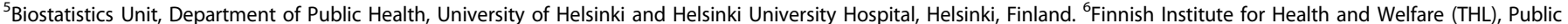

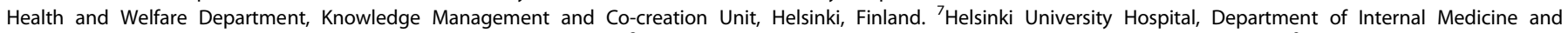

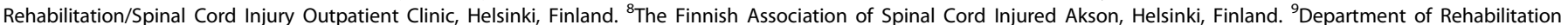

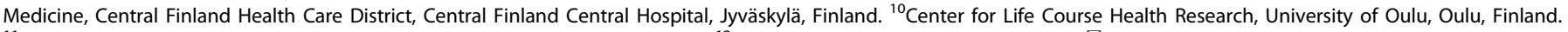

${ }^{11}$ The Finnish Association of People with Physical Disabilities, Helsinki, Finland. ${ }^{12}$ Validia Rehabilitation, Helsinki, Finland. ${ }^{凶}$ email: susannatallqvist@gmail.com
} 


\section{METHODS}

\section{Design}

FinSCl is a collaborative study among The Finnish Association of People with Physical Disabilities, The Finnish Association of Spinal Cord Injured Akson, The Finnish Institute for Health and Welfare (THL), and the $\mathrm{SCl}$ outpatient clinics of three university hospitals (Oulu, Tampere, and Helsinki). The catchment population and locations of these hospitals are presented in Supplement (Figure A). The purpose of the FinSCl is to identify the factors that are related to the health and functioning of persons with $\mathrm{SCl}$, their challenges with environmental factors of accessibility, and how such factors are interconnected [9].

\section{Sample}

Study participants were identified from the registers of the SCl outpatient clinics from the university hospitals in Oulu, Tampere, and Helsinki, where their clinical data were also collected. The inclusion criteria were as follows: age of at least 16 years; non-traumatic SCI (NTSCl) or traumatic SCI (TSCl); and AIS classification of A, B, C, or D. International Standards for Neurological Classification of SCI (ISNCSCI) was used to determine The American Spinal Injury Association Impairment Scale (AIS) [12]. Patients with AIS E, living in an institute, or having a congenital $\mathrm{SCl}$, progressive or neurodegenerative disease, multiple sclerosis, amyotrophic lateral sclerosis, or Guillain-Barre syndrome were excluded. The detailed protocol, the precise patient selection process, the ethical considerations, and the content of the formulated questionnaire have been presented in a separate publication [9]. The questionnaire was sent home to respondents in February 2019, and the answers were collected until the end of July 2019.

\section{Outcome measures}

A questionnaire from the National Survey of Health, Well-being, and Service (FinSote) was applied to screen 12 comorbidities [13] and the Spinal Cord Injury Secondary Condition Scale (SCl-SCS) was used to screen 16 SHCs [14]. The prevalence of multimorbidity was analysed separately for comorbidities and SHCs. FinSote and SCl-SCS are self-reported outcome measures in which the data are collected from patients/persons who are living with their health condition [15]. SCI-SCS is also used in the International Spinal Cord Injury Survey, InSCI [16], in which FinSCl is not involved due to differences in study schedules and partly different self-reported outcome measures. The FinSCl study uses the International Classification of Functioning, Disability, and Health (ICF) [17] as a theoretical framework, and this article focuses on the analyses of body functions categories and health conditions (Supplementary Table A).

FinSote is a survey that is performed by the THL. The data are collected in 10,000 randomly selected individuals aged 20 years or older annually, and in 60,000 individuals every four years [13]. The relevance of the statistical analyses and the description of the utilised methods have been comprehensively reported by the THL [18]. This article analysed the prevalence of 12 different comorbidities that were diagnosed or treated by a physician during the last 12 months. The THL provides open access data concerning the four most common comorbidities: high blood pressure/hypertension, spondylosis, sciatica or other spinal disease (later referred to as back problems), high cholesterol, and coronary heart disease (CHD) or angina pectoris. Comparisons between the population with $\mathrm{SCl}$ and the reference group were conducted among these four illnesses. Information on the presence of CHD was only requested from participants who were aged 54 or older in the FinSote survey [18]. This study used the FinSote 2018 data as a reference, and the age groups were reclassified for this study by the THL.

The SCI-SCS is a self-reported health instrument scale that is used by persons with $\mathrm{SCl}$, in which respondents are asked to rate their health over the last 3 months. The measure comprises 16 symptoms or conditions, 2 of which (diabetes mellitus and heterotopic ossification) should also be diagnosed by physicians. SHCs are evaluated on a scale from 0 to $3(0=$ not experienced in the last 3 months or is an insignificant problem; $1=$ mild or infrequent problem; 2 =moderate or occasional problem; and $3=$ significant or chronic problem), with a maximum score of 48 . The higher the score, the more health problems the respondent experiences [14, 19]. The SCI-SCS has demonstrated reliability and validity, and it is included in the Spinal Cord Injury Research Evidence database as a recommended health measure to be used for persons with SCI [20].

\section{Statistical analyses}

Duplicate responses were checked, and the second response was omitted There were few missing values in the data (0.4-4.5\% per question), in which case no imputation was performed. The data concerning the national reference group were received from the $\mathrm{THL}$, where the missing values were corrected by using the inverse probability weighting method (IPW). The IPW method calculates a weighting factor for each respondent by taking into account the following variables: age, gender, marital status, level of education, province of residence, and its population density.

Descriptive statistics for the general and lesion characteristics, and results are presented as means (and standard deviations), medians (and interquartile ranges), and frequencies (and percentages), as appropriate. To assess the generalisability of the results, an analysis between participants and nonparticipants was performed by chi-square tests. Chi-square tests were also used to analyse the between group comparisons among the general and lesion characteristics. $P$-values for the pairwise comparisons were adjusted by the use of Bonferroni correction. The Z-test from Epitools-Epidemiological Calculators were used to compare the proportions of the comorbidities between the reference population and the population with $\mathrm{SCl}$ [21]. Multimorbidity was evaluated as the number of reported comorbidities (scale range 0-12) and SHCs (scale range 0-16). Two separate negative binomial regression models were used to examine the associations between general and lesion characteristics, and comorbidities and SCHs. Negative binomial regression modelling was used to estimate unadjusted and adjusted incidence rate ratios for the general and lesion characteristics, taking into account the overdispersion in the multimorbidity count data. Additionally, 95\% confidence intervals (Cls) were used, and $p$-values of $<0.05$ were considered to be statistically significant. Statistical analyses were performed using SPSS version 25 .

\section{RESULTS}

\section{Characteristics of participants and non-respondents}

The response rate in the survey was $50 \%$, and the final number of participants was 884. Participants and non-respondents were categorised according to the recommendations of the International Spinal Cord Injury Core Data Set (Table 1) [12]. The youngest participant was 20 years old, which is why the first age group is 20-30 years. In the analyses between participants $(n=884)$ and nonrespondents $(n=888)$, there was a statistically significant difference between genders and age groups; younger persons aged 20-45 years answered less frequently, and persons $61-75$ years of age, as well as females, actively participated. Two participants used a ventilator and were included in group C1-4 AIS A, B, and C based on the recommendations of the International Spinal Cord Injury Core Data Set [12]. The shortest time since an injury was 11 months and this value was rounded up to one year. There was one transgender participant who was grouped by the gender according to the hospital records. An internal analysis of the participants indicated that group AIS D in all injury levels (later referred to as group AIS D) differed from the other severity of SCl groups (Supplementary Table B).

\section{Comorbidities}

There were 287 persons (33\%) with SCl who answered that they had none of the 12 comorbidities. The two most common comorbidities diagnosed or treated in more than $25 \%$ of the population with $\mathrm{SCl}$ in Finland during the last 12 months were high blood pressure/ hypertension and back problems (Fig. 1). The population with SCI had more of these problems than the reference population (Table 2a). The reference data were available only for the four most common comorbidities (high blood pressure/hypertension, back problems, high cholesterol, and CHD or angina pectoris). High cholesterol was the third most common comorbidity; in both populations it manifested in the same manner, but was more common among the reference population aged 61-75 years. In the subgroup analyses, $\mathrm{CHD}$ or angina pectoris was higher among the male reference group compared to males with $\mathrm{SCl}$, and no difference between female reference and $\mathrm{SCl}$ groups (Table $2 \mathrm{a}$ ).

Many comorbidities varied according to general characteristics among the population with $\mathrm{SCl}$ (Table $2 \mathrm{~b}$ ). There were more reports of back problems and asthma in females than in males. The incidences of high blood pressure/hypotension, high cholesterol, back problems, coronary heart disease or angina pectoris, and diabetes were lowest among the youngest participants and increased in every age group, being highest among persons aged $\geq 76$. Depression and 
Table 1. Eligible population of the Finnish Spinal Cord Injury study divided to participants (N884) and non-respondents (N888).

\begin{tabular}{|c|c|c|c|}
\hline & $\begin{array}{l}\text { Participants N884 } \\
\text { n (\%) }\end{array}$ & $\begin{array}{l}\text { Non-respondents N888 } \\
n(\%)\end{array}$ & $p$ value \\
\hline Gender & & & $<0.01$ \\
\hline Male & $577(65 \%)$ & $633(71 \%)$ & \\
\hline Aetiology & & & 0.10 \\
\hline Traumatic & 492 (56\%) & 527 (59\%) & \\
\hline C1-4 AIS A, B, and C & $95(12 \%)$ & $107(11 \%)$ & \\
\hline C5-8 AIS A, B, and C & $55(6 \%)$ & $62(7 \%)$ & \\
\hline T1-S5 AIS A, B, and C & $184(21 \%)$ & $209(24 \%)$ & \\
\hline AIS D at any injury level & $550(62 \%)$ & $510(57 \%)$ & \\
\hline Age, years & $\min 20, \max 90$, mean 61, SD 14 & $\min 17, \max 93$, mean 54, SD 17 & $<0.01$ \\
\hline $46-60$ & $238(27 \%)$ & $243(27 \%)$ & \\
\hline $61-75$ & $386(44 \%)$ & $243(27 \%)$ & \\
\hline$\geq 76$ & $118(13 \%)$ & $102(12 \%)$ & \\
\hline \multirow[t]{2}{*}{ Time since injury, years } & $\min 1, \max 67$, mean 11, SD 11 & $\min 1, \max 66$, mean 10, SD 10 & 0.52 \\
\hline & median 7 IQR 4-14 & median 6 IQR 4-14 & \\
\hline $1-5$ years & $353(40 \%)$ & 379 (43\%) & \\
\hline $6-10$ years & $227(26 \%)$ & $222(25 \%)$ & \\
\hline $11-15$ years & $128(14 \%)$ & $111(12 \%)$ & \\
\hline$\geq 16$ years & $176(20 \%)$ & $176(20 \%)$ & \\
\hline
\end{tabular}

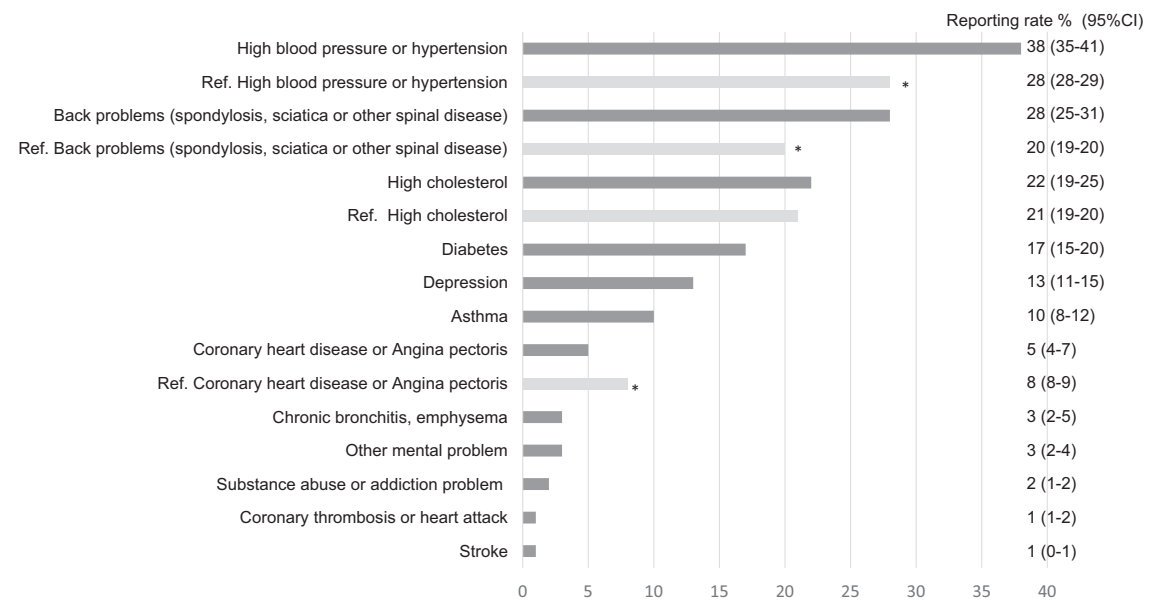

Distribution (\%) of persons reporting of a comorbidity diagnosed or treated by a physician during last 12 months (\%)

Note * $p<0.01$ between population with $\mathrm{SCl}$ and reference population

Comparisons were available in comorbidities; high blood pressure or hypertension, back problems, high cholesterol and coronary heart disease

or angina pectoris (see Outcome measures). Bar charts of reference population (Ref.) is marked with light grey.

Fig. 1 Prevalence of comorbidities (percentage with $95 \%$ confidence interval) diagnosed or treated by a physician during the last 12 months in the Finnish Spinal Cord Injury study (884 participants) and the reference population (Ref.).

substance abuse or addiction problems were most common in the 46-60 age group with a statistically significant difference in those aged 61-75 years. Other mental problems were more frequent in the youngest age group than in the three oldest groups.

Between severity of $\mathrm{SCl}$ groups there were statistically significant differences in high cholesterol, high blood pressure/hypertension, and back problems (Table 2b), but the differences in high cholesterol vanished in the pairwise analyses after Bonferroni correction. High blood pressure/hypertension was more frequent in group T1-S5 AIS $A, B$, and $C$, as well as in group AIS D, than in group C5-8 AIS A, B, and C. Back problems were most frequent in group AIS $D$ in comparison to all of the other groups. Additionally, there were some differences in 


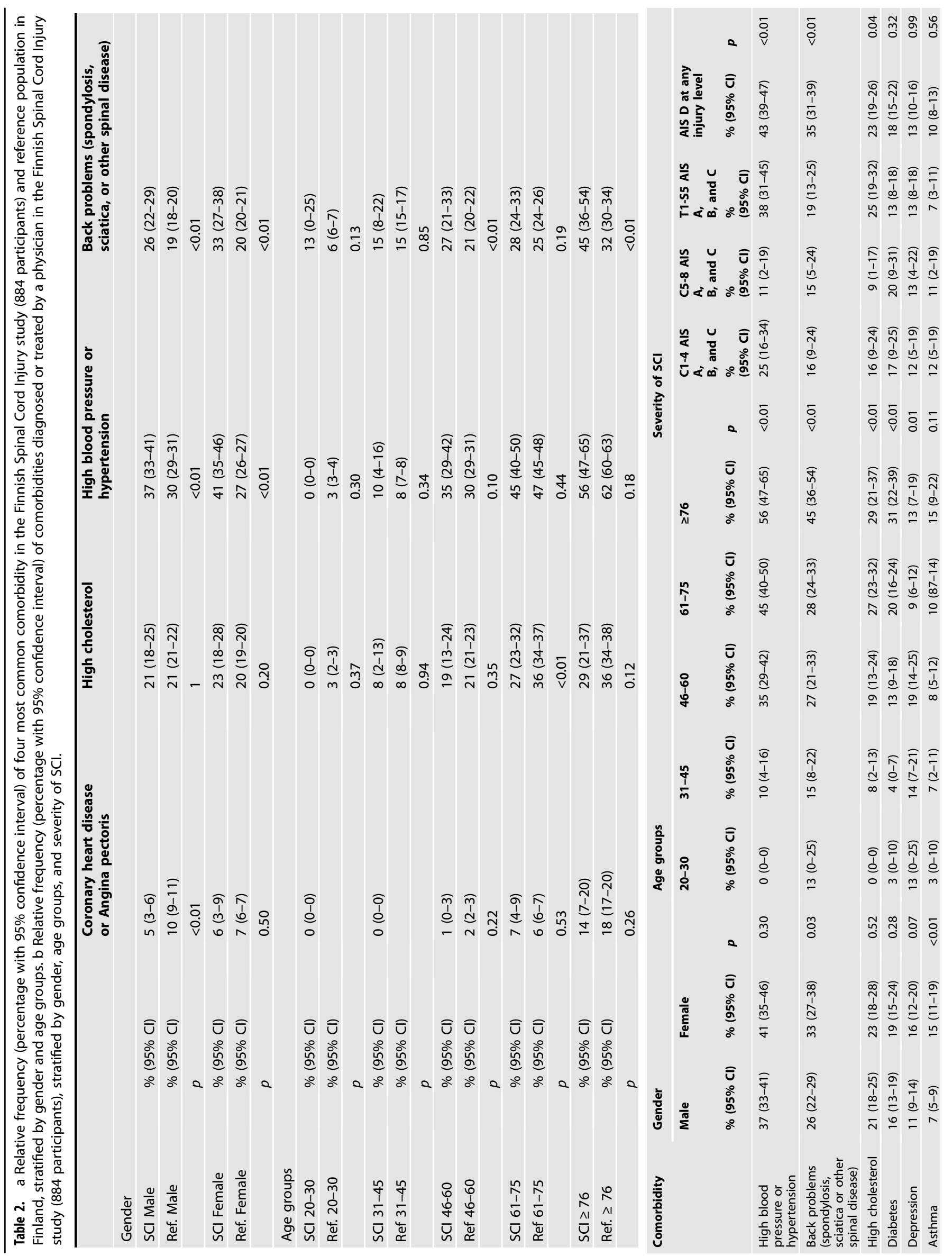


the prevalence of the comorbidities from the aspect of time since injury and aetilogy (Supplementary Table C).

\section{Secondary health conditions}

Over $70 \%$ of the Finnish population with $\mathrm{SCl}$ reported suffering at least mildly or infrequently from joint and muscle pain, muscle spasms (spasticity), chronic pain, and bowel dysfunction (Fig. 2). Twelve participants (1\%) reported no SHCs.

Males reported more sexual dysfunction, circulatory problems, and pressure sores than females. Bladder and bowel dysfunction, as well as joint and muscle pain were more frequent among females (Table 3). As in the comorbidities, several SHCs, such as heterotopic ossification, diabetes mellitus (included in the outcome of SHCs), respiratory problems, and contractures increased with age. Circulatory problems and autonomic dysreflexia differed between the age groups, but differences were not statistically significant after Bonferroni correction. Injuries due to loss of sensation, and chronic pain were highest among the 46-60 age group, with a statistically significant difference compared to the two oldest age groups (loss of sensation) and the 31-45 age group (chronic pain).

Autonomic dysreflexia was more common in groups C1-4 AIS A, B, and $C$ and $C 5-8$ AIS A, B, and C than in the two other groups (Table 3). Postural hypotension was most common in group C1-4 AIS A, B and $C$ with a statistically significant difference in comparison to groups T1S5 AIS A, B, C, and AIS D. Group T1-S5 AIS A, B, C had fewer respiratory problems than other groups. Spasticity was the most common in group C1-4 AIS A, B, and C, and the difference was statistically significant in comparison to groups T1-S5 AIS A, B, and C and AIS D. Sexual dysfunction, circulatory problems as well as bowel and bladder dysfunctions were the most common in group T1-S5 AIS A, B and C, and the differences were statistically significant compared to group AIS D. Group AIS D had fewer pressure sores and urinary tract infections than all of the other $\mathrm{SCl}$ severity groups. Contractures differed in the severity of $\mathrm{SCl}$ groups, but this difference was not statistically significant in the pairwise comparisons after Bonferroni correction (Table 3). Additionally, there were some differences in the prevalence of the SHCs with regard to time since injury and aetilogy (Supplementary Table D).

\section{Multimorbidity}

The mean number of reported comorbidities varied between the general and lesion characteristics (Table 4). The mean was lowest in the 20-30 years age group (0.48) and was highest among the participants aged 76 years or older (2.0). The scale range for the comorbidities was $0-12$. For the SHCs, the lowest mean was also observed among the youngest participants (6.4), but the highest mean was observed in the SCl severity group C1-4 AIS A, B, and C (8.9). The scale range for the SHCs was $0-16$. Statistically significant differences were found in the comorbidities between the age groups, in which the expected number increased with age (being $338 \%$ higher in the oldest age group than in the youngest). Persons with NTSCl had a $27 \%$ higher expected number of comorbidities than persons with $\mathrm{TSCl}$ in the adjusted model (Table 4).

\section{DISCUSSION}

This study assessed the comorbidities and SHCs among the Finnish population with $\mathrm{SCl}$. Analyses of the comorbidities suggested that high blood pressure/hypertension and back problems were more common in the population with $\mathrm{SCl}$ than among the reference population, as was suspected from previous studies [3, 4]. However, $\mathrm{CHD}$ or angina pectoris was more frequent in the male reference population than in the male population with $\mathrm{SCl}$. This result is contradictory in comparison to international studies which indicate that individuals with $\mathrm{SCl}$ are known to have a higher risk for cardiovascular diseases [22, 23]. Since it is also known that cardiovascular disease is one of the general chronic conditions among the Finnish population [24], our result raises a question of 


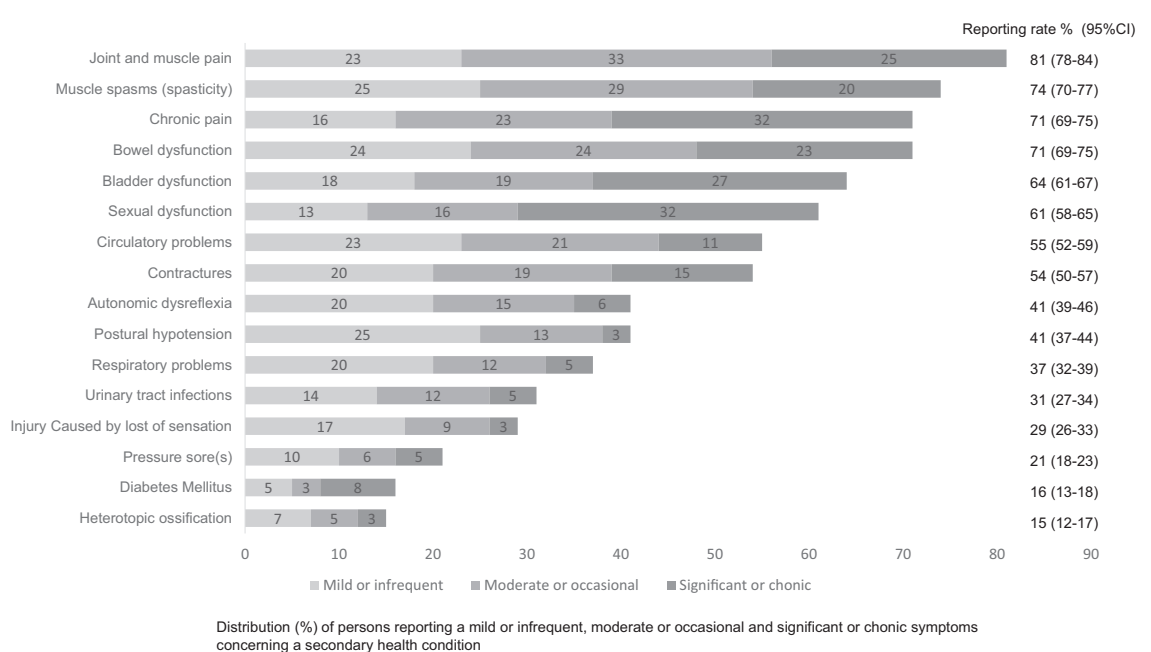

Fig. 2 Prevalence of secondary health conditions (percentage with $95 \%$ confidence interval) in the Finnish Spinal Cord Injury study (884 participants).

whether $\mathrm{CHD}$ is underdiagnosed among males with $\mathrm{SCl}$ in Finland. Thus, more attention should be paid to the possibility of this disease.

Ageing was a prominent factor in elevating the relative risk of multimorbidity in the analyses of the comorbidities among the population with $\mathrm{SCl}$ in Finland. Similar results have been reported in other studies [4, 25], and this can be considered as a natural phenomenon since ageing leads to an increase in morbidities [26]. This study suggests that the time since injury (i.e., ageing with $\mathrm{SCl}$ ) is not associated with multimorbidity and that elderly persons with $\mathrm{SCl}$ often have other comorbidities as well. In Finland, the incidence of $\mathrm{SCl}$ is at 92 per million inhabitants annually; $60 \%$ of these are $\mathrm{NTSCl}$, and their mean age is 62 years $[11,27]$.

As expected, SHCs following $\mathrm{SCl}$ were often experienced by persons with cervical lesions classified by ISNCSCl as AIS A, B, and C. Higher injury level and more complete injury are related to a higher rate of medical complications [4-6, 8]. However, in the analyses of the relative risk of multimorbidity, there were no statistically significant differences between the severity of $\mathrm{SCl}$ groups. This result is contradictory, as Brinkhof et al. [5] indicated that persons with complete tetraplegia have a higher expected number for multimorbidity, as measured by a modified SCI-SCS. In their study, also persons with NTSCI had a higher relative risk for SHCs compared to persons with $\mathrm{TSCl}$, which is different from our result. Regardless, the aetiology was a relevant indicator of multimorbidity in the analyses of the comorbidities in this study, since persons with NTSCI had a higher expected number of comorbidities than persons with TSCl.

As a single symptom, pain is one of the top three most frequent SHCs in several countries $[5,8,28,29]$, including Finland. In the present study, pain (chronic or joint/muscle) was experienced equally among all the characteristics. The negative influences that are associated with pain, such as depressive symptoms, more restricted and less satisfying participation, and low quality of life, can affect persons with $\mathrm{SCl}$ [28]. Unfortunately, number of persons with $\mathrm{SCl}$ who report pain problems has remained almost the same over the last decades [30].

\section{Strengths and limitations}

As a benefit to the FinSCl study, from the planning stage to the realisation of this study, we have observed the active participation of persons with $\mathrm{SCl}$ with assistance from The Finnish Association of Spinal Cord Injured Akson. We believe that this has increased the response rate. Another advantage of this study was the access to the registered $\mathrm{SCl}$ outpatient clinic data, which made it possible to identify the majority of persons with $\mathrm{SCl}$ in Finland, and to reliably combine their general and lesion characteristics. However, the fact that the ISNCSCI classifications were completed by several physicians and physiotherapists between 2000 and 2018 has to be recognised. Even so, the protocol of the ISNCSCI was followed, and the latest classification from the medical records was used.

This study had several limitations. For example, a portion of the data were skewed; thus the results must be interpreted with caution. The high incidence of individuals in the $\mathrm{SCl}$ severity group AIS D was expected, since approximately $65 \%$ of the population with $\mathrm{SCl}$ in Finland belong to this group [11]. Group AIS D differed significantly from the other $\mathrm{SCl}$ severity groups because there were more elderly and non-traumatically injured persons, and most of the participants were injured within the last five years. Additionally, the younger population with $\mathrm{SCl}$ did not answer the survey as actively as the older individuals. The age and gender structure of the target population for FinSote and FinSCl are different and this aspect needs to be noted when interpreting the results. The national reference data were available for only four comorbidities, which makes these analyses incomplete. The data were collected by self-reported outcome measures, and the veracity of the comorbidities that were diagnosed or treated by a physician was not checked in the hospital records, which may have caused some inaccuracy in the data.

\section{CONCLUSIONS AND FUTURE DIRECTIONS}

The prevalence of comorbidities and SHCs was common among elderly persons with $\mathrm{SCl}$. We found that the strongest predictor for multimorbidity was age. This finding emphasises the importance of geriatric-related knowledge in $\mathrm{SCl}$ because the number of senior citizens, the life expectancy of persons with $\mathrm{SCl}$, and the incidence of SCls will increase in the future $[10,11]$. This can then cause a greater burden on Finland's health care system, and it is necessary to create sufficient healthcare services with expertise in $\mathrm{SCl}$. Additionally, possible preventive actions to minimise the risks of injuries should be evaluated early enough.

Persons with the most severe disabilities (that is, $\mathrm{SCl}$ severity groups C1-4 AIS A, B and C and C5-8 AIS A, B, and C) also reported a high prevalence of comorbidities and SHCs, even though there was no statistically significant difference between the severity of $\mathrm{SCl}$ groups. Additionally, persons with NTSCl had a higher risk of the multimorbidity than those with TSCl in the analyses of the comorbidities. Thus, we believe that the needs of persons with cervical lesions AIS A, $B$, or $C$, and persons with NTSCl should also receive more attention in future $\mathrm{SCl}$ research.

We found that the prevalence of SHCs in the Finnish population with $\mathrm{SCl}$ was high and that pain (as a single symptom) was consistently one of the main problems in their daily lives. Therefore, the regular evaluation of SHCs, especially pain, and 


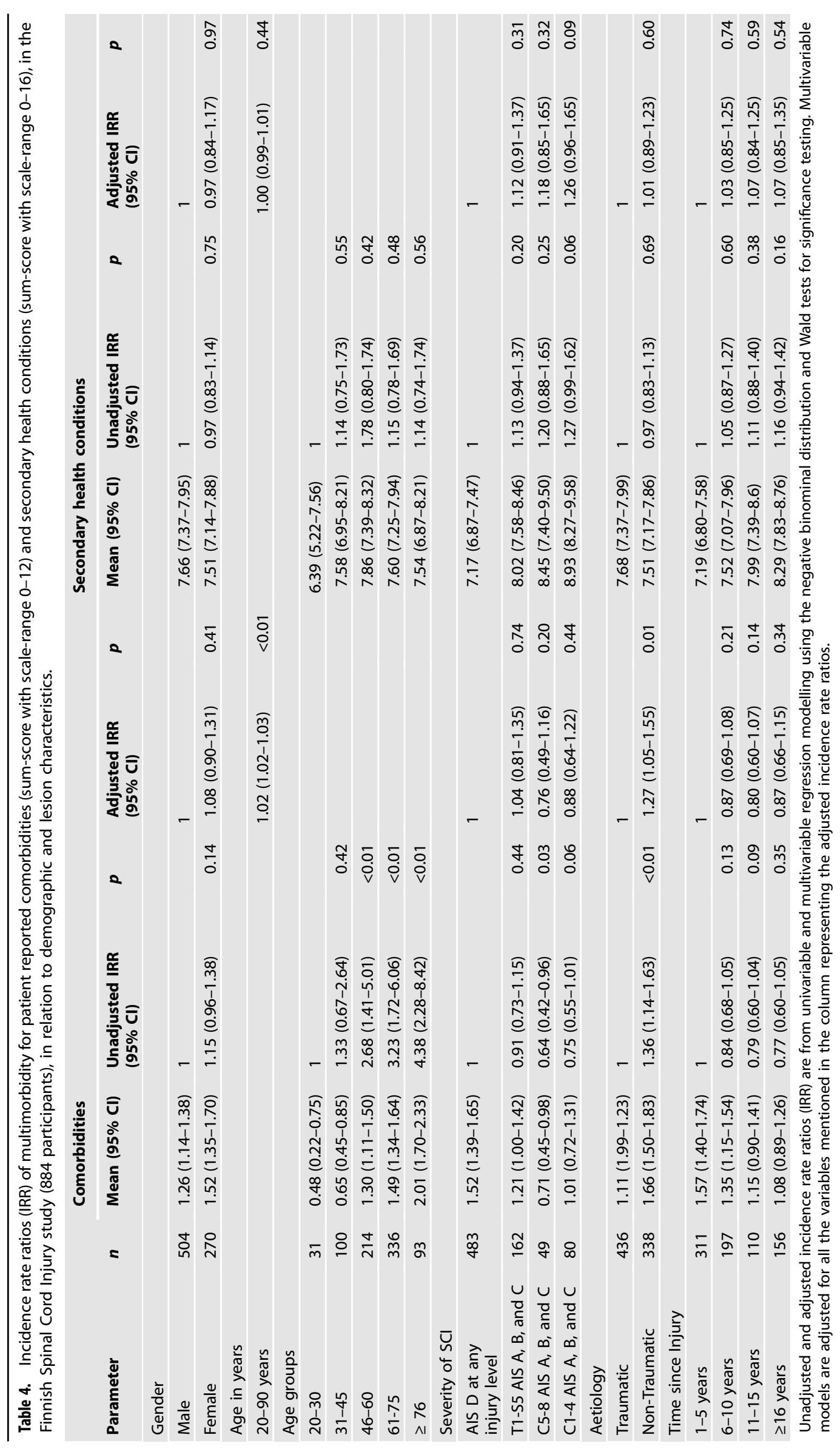


the effective use of pharmacological and nonpharmacological methods to ease SHCs are extremely important.

This study suggests that in Finland, the population with $\mathrm{SCl}$ has more comorbidities than the reference population. Further research is needed to assess this issue in more detail.

\section{DATA ARCHIVING}

The authors will consider any reasonable requests to access the data.

\section{REFERENCES}

1. Valderas JM, Starfield B, Sibbald B, Salisbury C, Roland M. Defining comorbidity: implications for understanding health and health services. Ann Fam Med. 2009;7:357-63.

2. Jensen MP, Molton IR, Groah SL, Campbell ML, Charlifue S, Chiodo A, et al. Secondary health conditions in individuals aging with $\mathrm{SCl}$ : terminology, concepts and analytic approaches. Spinal cord. 2011;50:373-8.

3. Tate DG, Charlifue S, Forchheimer M, Jay G, Wingo BC, Requejo PS, et al. Comorbidities and age related conditions among persons with spinal cord. Inj/ Dis (SCI/D) ARCH PHYS MED REHAB. 2015;96:e49-e49.

4. Marion TE, Rivers CS, Kurban D, Cheng CL, Fallah N, Batke J, et al. Previously identified common post-injury adverse events in traumatic spinal cord injury-validation of existing literature and relation to selected potentially modifiable comorbidities: a prospective Canadian cohort study. J Neurotrauma. 2017;34:2883-91.

5. Brinkhof MW, Al-Khodairy A, Eriks-Hoogland I, Fekete C, Hinrichs T, HundGeorgiadis $\mathrm{M}$, et al. Health conditions in people with spinal cord injury: contemporary evidence from a population-based community survey in Switzerland. J Rehabil Med. 2016;48:197-209.

6. Craven C, Hitzig SL, Mittmann N. Impact of impairment and secondary health conditions on health preference among Canadians with chronic spinal cord injury. J Spinal Cord Med. 2012;35:361-70.

7. Engel L, Bryan S, Noonan VK, Whitehurst DGT. Using path analysis to investigate the relationships between standardized instruments that measure health-related quality of life, capability wellbeing and subjective wellbeing: an application in the context of spinal cord injury. Soc Sci Med. 2018;213:154-64.

8. Adriaansen JJ, Post MW, de Groot S, van Asbeck FW, Stolwijk-Swuste JM, Tepper M, et al. Secondary health conditions in persons with spinal cord injury: a longitudinal study from one to five years post-discharge. J Rehabil Med. 2013;45:1016-22.

9. Tallqvist $S$, Anttila $H$, Kallinen $M$, Koskinen $E$, Hämäläinen $H$, Kauppila A-M, et al. Health, functioning and accessibility among spinal cord injury population in Finland: protocol for the FinSCI study. J Rehabil Med. 2019;51:273-80.

10. Groah SL, Charlifue S, Tate D, Jensen MP, Molton IR, Forchheimer M, et al. Spinal cord injury and aging: challenges and recommendations for future research. Am J Phys Med Rehabil. 2012;91:80-93.

11. Koskinen $E$, Väärälä $E$, Alen $M$, Kallinen $M$, Vainionpää $A$. Selkäydinvammojen ilmaantuvuus on ennakoitua suurempi [Incidence of spinal cord injuries in Finland higher than expected]. Lääkärilehti. 2017;39:2160-2165b.

12. Biering-Sorensen F, DeVivo MJ, Charlifue S, Chen Y, New PW, Noonan V, et al. International Spinal Cord Injury Core Data Set (version 2.0)-including standardization of reporting. Spinal Cord. 2017;55:759-64.

13. Finnish Institute of Health and Welfare. National FinSote Survey 2017; Available at: https://thl.fi/en/web/thlfi-en/research-and-expertwork/population-studies/ national-finsote-survey. Accessed [Cited Sep 10], 2020.

14. Kalpakjian CZ, Scelza WM, Forchheimer MB, Toussaint LL. Preliminary reliability and validity of a spinal cord injury secondary conditions scale. J Spinal Cord Med. 2007;30:131-9.

15. Coulter A. Measuring what matters to patients. BMJ. 2017;356:j816.

16. Gross-Hemmi MH, Post MW, Ehrmann C, Fekete C, Hasnan N, Middleton JW, et al. Study protocol of the International Spinal Cord Injury (InSCI) community survey. Am J Phys Med Rehabil. 2017;96:S23-S34.

17. WHO. International Classification of Functioning, Disability and Health (ICF). Geneva: World Health Organization; 2001.

18. Pentala-Nikulainen $\mathrm{O}$, Koskela $\mathrm{T}$, Parikka $\mathrm{S}$, Kilpeläinen $\mathrm{H}$, Koskenniemi T, Aalto A$M$, et al. Kansallisen terveys-, hyvinvointi ja palvelututkimus FinSoten perustulokset 2017-8. [The Results of the National survey of health, well-being and service, Finsote survey 2017-8]. Available at: thl.fi/finsote.

19. Arora M, Harvey LA, Lavrencic L, Bowden JL, Nier L, Glinsky JV, et al. A telephonebased version of the spinal cord injury-secondary conditions scale: a reliability and validity study. Spinal Cord. 2016;54:402-5.

20. Spinal Cord Injury Research Evidence. Spinal Cord Injury Secondary Conditions Scale (SCI-SCS). 2018; Available at: https://scireproject.com/outcome-measures/ outcome-measure-tool/spinal-cord-injury-secondary-conditions-scale-sci-scs/. Accessed [Cited Sep 10], 2020.
21. Sergeant E. Epitools Epidemiological Calculators. 2018; Available at: http:// epitools.ausvet.com.au.

22. Bauman WA, Spungen AM. Coronary heart disease in individuals with spinal cord injury: assessment of risk factors. Spinal Cord. 2008;46:466-76.

23. Cragg JJ, Noonan VK, Krassioukov A, Borisoff J. Cardiovascular disease and spinal cord injury: results from a national population health survey. Neurology. 2013:81:723-8.

24. Finnish Institute for Health and Welfare. Chronic diseases. 2020; Available at: https://thl.fi/en/web/chronic-diseases. Accessed [Cited Sep 10], 2020.

25. Saunders LL, Clarke A, Tate DG, Forchheimer M, Krause JS. Lifetime prevalence of chronic health conditions among persons with spinal cord injury. Arch Phys Med Rehabil. 2015;96:673-9.

26. Fabbri E, Zoli M, Gonzalez-Freire M, Salive ME, Studenski SA, Ferrucci L. Aging and multimorbidity: new tasks, priorities, and frontiers for integrated gerontological and clinical research. J Am Med Dir Assoc. 2015;16:640-7.

27. Niemi-Nikkola V, Koskinen $E$, Väärälä $E$, Kauppila $A M$, Kallinen $M$, Vainionpää $A$. Incidence of acquired nontraumatic spinal cord injury in Finland: a 4-year prospective multicenter study. Arch Phys Med Rehabil. 2021;102:44-49.

28. Mittmann N, Hitzig SL, Craven CB. Predicting health preference in chronic spinal cord injury. J Spinal Cord Med. 2014;37:548-55.

29. New PW. Secondary conditions in a community sample of people with spinal cord damage. J Spinal Cord Med. 2016;39:665-70.

30. Cardenas DD, Bryce TN, Shem K, Richards JS, Elhefni H. Gender and minority differences in the pain experience of people with spinal cord injury. Arch Phys Med Rehabil. 2004;85:1774-81.

\section{ACKNOWLEDGEMENTS}

We want to thank researcher Suvi Parikka and statistical researcher Jonna lkonen from the Finnish Institute for Health and Welfare for providing and reclassifying the reference data; PT, MSc student Kirsi Majamäki for helping enter written answers into Webropol; PT, MSc, $\mathrm{PhD}$ student Joonas Poutanen from the University of Helsinki/Validia Rehabilitation for all the comments and feedback; and graphic designer Johanna Paulin from The Finnish Association of People with Physical Disabilities for designing the Supplementary Figure A.

\section{AUTHOR CONTRIBUTIONS}

Sinikka Hiekkala was the principal investigator and director of the entire FinSCl study. Susanna Tallqvist collected the participants' data from the registers of the SCl outpatient clinics. Susanna Tallqvist and Sinikka Hiekkala formulated the enquiry, and all authors as well as seven persons with $\mathrm{SCl}$ provided feedback on it. All authors participated in designing and providing feedback on the report. Susanna Tallqvist analysed the data, interpreted the results and wrote the report.

\section{FUNDING}

This study was supported by the Finnish Association of People with Physical Disabilities (pr42105, 2017-2020); Oulu University Hospital (grant VTR K86709, 20.10.2017); Department of Internal Medicine and Rehabilitation, Helsinki University Hospital (grants HUS/53/2017 §9, 9.6.2017, HUS/76/2018 § 11, 18.4.2018 and HUS/174/201 §1, 12.4.2019); and Validia Rehabilitation (grant HUS-VTR 9.3.2017). Open access funding provided by University of Helsinki including Helsinki University Central Hospital.

\section{COMPETING INTERESTS}

The authors declare no competing interests.

\section{STATEMENT OF ETHICS}

The study was approved by the HUS Coordinating Ethics Committee (HUS/1776/2017).

\section{ADDITIONAL INFORMATION}

Supplementary information The online version contains supplementary material available at https://doi.org/10.1038/s41393-021-00704-7.

Correspondence and requests for materials should be addressed to Susanna Tallqvist.

Reprints and permission information is available at http://www.nature.com/ reprints

Publisher's note Springer Nature remains neutral with regard to jurisdictional claims in published maps and institutional affiliations. 
Open Access This article is licensed under a Creative Commons Ac Attribution 4.0 International License, which permits use, sharing,
adaptation, distribution and reproduction in any medium or format, as long as you give appropriate credit to the original author(s) and the source, provide a link to the Creative Commons license, and indicate if changes were made. The images or other third party material in this article are included in the article's Creative Commons license, unless indicated otherwise in a credit line to the material. If material is not included in the article's Creative Commons license and your intended use is not permitted by statutory regulation or exceeds the permitted use, you will need to obtain permission directly from the copyright holder. To view a copy of this license, visit http://creativecommons. org/licenses/by/4.0/.

(c) The Author(s) 2021 